Acta Crystallographica Section E

Structure Reports

Online

ISSN 1600-5368

\section{T. Seethalakshmi, ${ }^{a}$}

S. Manivannan, ${ }^{\mathrm{a}}$ Daniel E. Lynch, ${ }^{b}$ S. Dhanuskodi ${ }^{\mathrm{a}}$ and P. Kaliannan ${ }^{\mathrm{a} *}$

${ }^{\text {a }}$ School of Physics, Bharathidasan University, Tiruchirappalli 620 024, India, and ${ }^{\mathbf{b}}$ Faculty of Health and Life Sciences, Coventry University, Coventry, CV1 5FB, UK

Correspondence e-mail: kal_44in@yahoo.co.in

\section{Key indicators}

Single-crystal X-ray study

$T=120 \mathrm{~K}$

Mean $\sigma(\mathrm{C}-\mathrm{C})=0.002 \AA$

$R$ factor $=0.021$

$w R$ factor $=0.052$

Data-to-parameter ratio $=21.1$

For details of how these key indicators were automatically derived from the article, see http://journals.iucr.org/e.

\title{
1-Ethyl-4-hydroxy-2,6-dimethylpyridinium bromide dihydrate
}

The title compound, $\mathrm{C}_{9} \mathrm{H}_{14} \mathrm{NO}^{+} \mathrm{Br}^{-} \cdot 2 \mathrm{H}_{2} \mathrm{O}$, comprises 1-ethyl2,6-dimethyl-4-hydroxypyridinium cations and bromide anions, with two solvent water molecules per formula unit. In the crystal structure, the anions, cations and water molecules are linked via intermolecular $\mathrm{O}-\mathrm{H} \cdots \mathrm{Br}$ and $\mathrm{O}-$ $\mathrm{H}$. . O hydrogen bonds, forming layers parallel to the (100) plane.

\section{Comment}

2,6-Dimethyl-4-hydroxypyridinone and 4-hydroxypyridinium salts have attracted much attention in the field of non-linear optics (NLO), since the 4-hydroxypyridinium conjugated electronic system could be an interesting hyperpolarizable chromophore for NLO activity (Manivannan et al., 2004; Dhanuskodi et al., 2006). To achieve self-assembly of organic cations in the manner required to exhibit NLO activity (Tamuly et al., 2005), suitable anions must be identified and used effectively. Halide anions have been reported to improve the physicochemical stability of 1-ethyl-2,6-dimethyl-4-(1H)pyridinones (Dhanuskodi et al., 2006). We report here the crystal structure of 1-ethyl-2,6-dimethyl-4-hydroxypyridinium bromide dihydrate (EDMPBr $\left.2 \mathrm{H}_{2} \mathrm{O}\right)$, (I).<smiles>CC[n+]1c(C)cc(O)cc1C</smiles>

(I)

The crystal structure of (I) (Fig. 1) comprises 1-ethyl-2,6dimethyl-4-hydroxypyridinium cations and bromide anions, with two solvent water molecules per formula unit. The $\mathrm{C} 2-$ $\mathrm{N} 1-\mathrm{C} 6$ bond angle in the cation $\left[120.71(16)^{\circ}\right]$ is comparable to that in 2,6-dimethylpyridine (Bond \& Davies, 2001) and the 2,6-dimethylpyridine-urea complex (Lee \& Wallwork, 1965). The organic cations lie in layers parallel to the (100) plane (Fig. 2). The bromide anions and water molecules lie between these layers, forming hydrogen-bonded sheets via $\mathrm{O}-\mathrm{H} \cdots \mathrm{O}$ and $\mathrm{O}-\mathrm{H} \cdots \mathrm{Br}$ interactions (Fig. 3 and Table 1). Two distinct ring motifs exist within these sheets, with graph-set descriptors $R_{4}^{6}(12)$ and $R_{10}^{6}(20)$ (Bernstein et al., 1995). O-H $\cdots \mathrm{O}$ hydrogen bonds are formed between atom O1 of the hydroxyl
Received 8 December 2006 Accepted 3 January 2007 


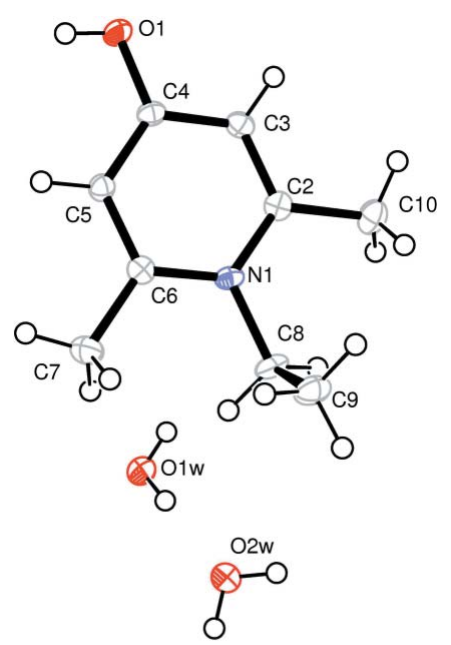

Figure 1

The molecular structure of (I), showing displacement ellipsoids drawn at the $50 \%$ probability level. $\mathrm{H}$ atoms are represented by circles of arbitrary radius.

group of the organic cation and one of the solvent water molecules (Fig. 2 and Table 1).

\section{Experimental}

The title compound was synthesized by dissolving 1-ethyl-2,6-dimethyl-4(1H)-pyridinone trihydrate (EDMP-3 $\mathrm{H}_{2} \mathrm{O}, 1.51 \mathrm{~g}$ ) with $\mathrm{HBr}$ $(2.43 \mathrm{~g})$ in distilled water $(5 \mathrm{ml})$. The solution was stirred well at room temperature for $7 \mathrm{~h}$ and the solvent was allowed to evaporate at $323 \mathrm{~K}$. The residual crystalline powder was redissolved in distilled water, and single crystals of (I) were obtained by slow evaporation at $303 \mathrm{~K}$.

\section{Crystal data \\ $\mathrm{C}_{9} \mathrm{H}_{14} \mathrm{NO}^{+} \mathrm{Br}^{-} \cdot 2 \mathrm{H}_{2} \mathrm{O}$ \\ $M_{r}=268.15$ \\ Monoclinic, $P 2_{1} / c$ \\ $a=10.5747(3) \AA$ \\ $b=8.0382(1) \AA$ \\ $c=15.0377$ (4) $\AA$ \\ $\beta=109.298(1)^{\circ}$ \\ $V=1206.41(5) \AA^{3}$}

$Z=4$

$D_{x}=1.476 \mathrm{Mg} \mathrm{m}^{-3}$

Mo $K \alpha$ radiation

$\mu=3.39 \mathrm{~mm}^{-1}$

$T=120$ (2) K

Block, colourless

$0.54 \times 0.48 \times 0.12 \mathrm{~mm}$

\section{Data collection}

Nonius KappaCCD diffractometer $\varphi$ and $\omega$ scans

Absorption correction: multi-scan (SADABS; Sheldrick, 2003)

$T_{\min }=0.176, T_{\max }=0.666$

\section{Refinement}

Refinement on $F^{2}$

$R\left[F^{2}>2 \sigma\left(F^{2}\right)\right]=0.021$

$w R\left(F^{2}\right)=0.052$

$S=1.04$

2768 reflections

131 parameters

$\mathrm{H}$-atom parameters constrained
15899 measured reflections 2768 independent reflections

2455 reflections with $I>2 \sigma(I)$

$R_{\text {int }}=0.033$

$\theta_{\max }=27.5^{\circ}$

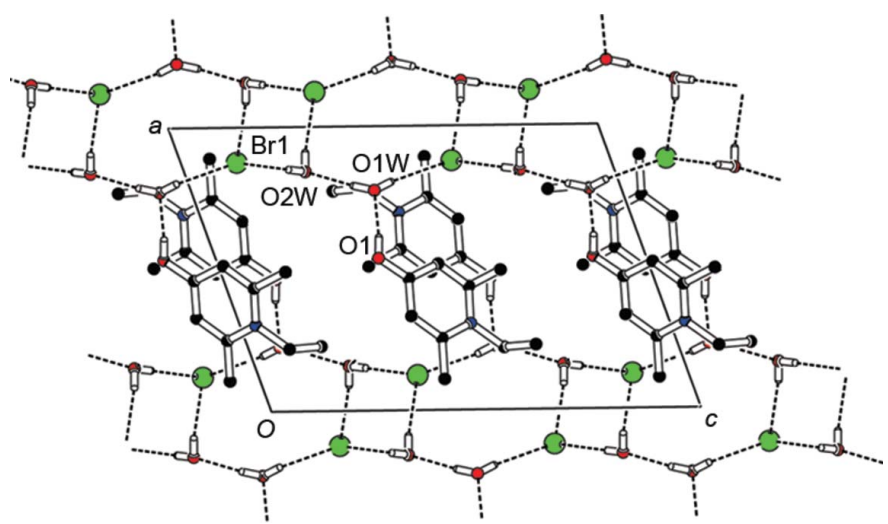

Figure 2

View of (I) along $b$, showing layers of organic cations lying parallel to the (100) plane, with $\mathrm{Br}^{-}$anions and water molecules lying between them. Dashed lines indicate hydrogen bonds. $\mathrm{H}$ atoms not involved in hydrogen bonding have been omitted.

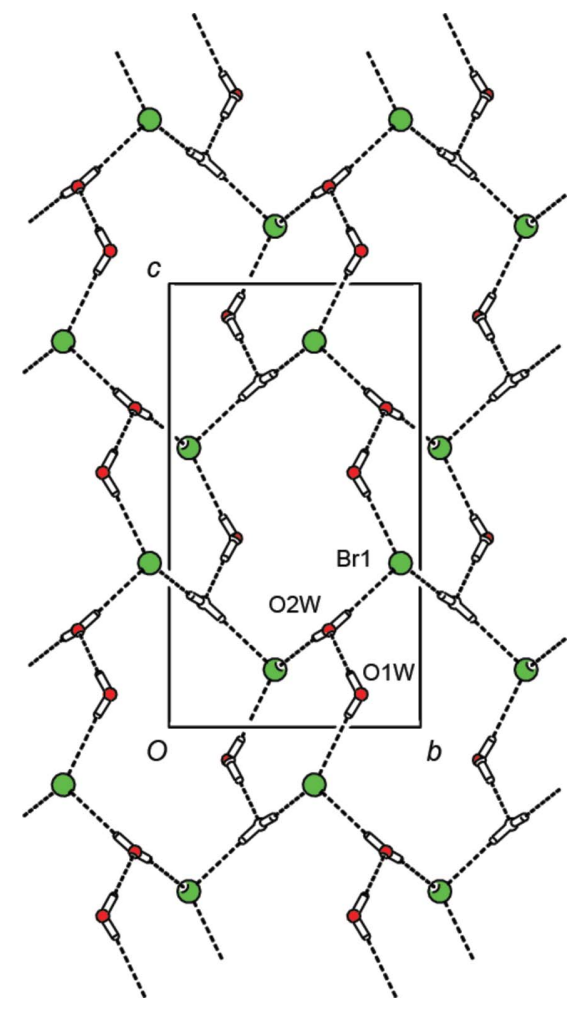

Figure 3

A single $\mathrm{Br}^{-} / \mathrm{H}_{2} \mathrm{O}$ sheet viewed along the $a$-axis direction, showing $\mathrm{O}-$ $\mathrm{H} \cdots \mathrm{O}$ and $\mathrm{O}-\mathrm{H} \cdots \mathrm{Br}$ hydrogen bonds (dashed lines).

Table 1

Hydrogen-bond geometry $\left(\AA,^{\circ}\right)$.

\begin{tabular}{lllll}
\hline$D-\mathrm{H} \cdots A$ & $D-\mathrm{H}$ & $\mathrm{H} \cdots A$ & $D \cdots A$ & $D-\mathrm{H} \cdots A$ \\
\hline $\mathrm{O} 1-\mathrm{H} 1 \cdots \mathrm{O} 1 W^{\mathrm{i}}$ & 0.80 & 1.78 & $2.5720(16)$ & 173 \\
$\mathrm{O} 1 W-\mathrm{H} 11 \cdots \mathrm{O} 2 W$ & 0.82 & 1.90 & $2.7135(16)$ & 175 \\
$\mathrm{O} 1 W-\mathrm{H} 12 \cdots \mathrm{Br} 1^{\text {ii }}$ & 0.76 & 2.50 & $3.2610(12)$ & 172 \\
$\mathrm{O} 2 W-\mathrm{H} 21 \cdots \mathrm{Br} 1$ & 0.81 & 2.52 & $3.3332(12)$ & 178 \\
$\mathrm{O} 2 W-\mathrm{H} 22 \cdots \mathrm{Br} 1^{\text {iii }}$ & 0.79 & 2.52 & $3.3061(12)$ & 173 \\
\hline
\end{tabular}

Symmetry codes: (i) $-x+1,-y+1,-z$; (ii) $x,-y+\frac{3}{2}, z-\frac{1}{2}$; (iii) $-x, y-\frac{1}{2},-z+\frac{1}{2}$. 
$\mathrm{H}$ atoms, except those of the water molecules, were positioned geometrically with $\mathrm{C}-\mathrm{H}=0.93(\mathrm{CH}), 0.96\left(\mathrm{CH}_{3}\right)$ or $0.97 \AA\left(\mathrm{CH}_{2}\right)$, and with $\mathrm{O}-\mathrm{H}=0.80 \AA$. They were then refined as riding, with $U_{\text {iso }}(\mathrm{H})=1.2 U_{\text {eq }}(\mathrm{C}, \mathrm{O})$ or $1.5 U_{\text {eq }}$ (methyl $\left.\mathrm{C}\right)$. $\mathrm{H}$ atoms of the water molecules were found in difference Fourier maps and refined initially with a restrained geometry. In the final cycles of refinement, they were made to ride on their parent $\mathrm{O}$ atoms, with $U_{\text {iso }}(\mathrm{H})=1.2 U_{\text {eq }}(\mathrm{O})$.

Data collection: COLLECT (Nonius, 1998); cell refinement: DENZO (Otwinowski \& Minor, 1997); data reduction: DENZO; program(s) used to solve structure: SIR92 (Altomare et al., 1994); program(s) used to refine structure: SHELXL97 (Sheldrick, 1997); molecular graphics: ORTEP-3 for Windows (Farrugia, 1997) and PLATON (Spek, 2003); software used to prepare material for publication: SHELXL97 and PARST (Nardelli 1995).

The authors thank the EPSRC National Crystallography Service (University of Southampton, UK) for the X-ray data collection. TS thanks Professor V. Parthasarathi, School of Physics, Bharathidasan University, for fruitful discussions.

\section{References}

Altomare, A., Cascarano, G., Giacovazzo, C., Guagliardi, A., Burla, M. C., Polidori, G. \& Camalli, M. (1994). J. Appl. Cryst. 27, 435.

Bernstein, J., Davis, R. E., Shimoni, L. \& Chang, N.-L. (1995). Angew. Chem. Int. Ed. Engl. 34, 1555-1573.

Bond, A. D. \& Davies, J. E. (2001). Acta Cryst. E57, o1039-o1040.

Dhanuskodi, S., Manivannan, S. \& Kirschbaum, K. (2006). Spectrochim. Acta Part A, 64, 504-511.

Farrugia, L. J. (1997). J. Appl. Cryst. 30, 565.

Lee, J. D. \& Wallwork, S. C. (1965). Acta Cryst. 19, 311-313.

Manivannan, S., Tiwari, S. K. \& Dhanuskodi, S. (2004). Solid State Commun. 132, 123-127.

Nardelli, M. (1995). J. Appl. Cryst. 28, 659.

Nonius (1998). COLLECT. Nonius BV, Delft, The Netherlands.

Otwinowski, Z. \& Minor, W. (1997). Methods in Enzymology, Vol. 276, Macromolecular Crystallography, Part A, edited by C. W. Carter Jr and R. M. Sweet, pp. 307-326. New York: Academic Press.

Sheldrick, G. M. (1997). SHELXL97. University of Göttingen, Germany. Sheldrick, G. M. (2003). SADABS. Version 2.10. Bruker AXS Inc., Madison, Wisconsin, USA.

Spek, A. L. (2003). J. Appl. Cryst. 36, 7-13.

Tamuly, C., Sarma, R. S., Batsanov, A. S., Goeta, A. E. \& Baruah, J. B. (2005). Acta Cryst. C61, o324-o327. 


\title{
supporting information
}

Acta Cryst. (2007). E63, o599-o601 [https://doi.org/10.1107/S1600536807000232]

\section{1-Ethyl-4-hydroxy-2,6-dimethylpyridinium bromide dihydrate}

\author{
T. Seethalakshmi, S. Manivannan, Daniel E. Lynch, S. Dhanuskodi and P. Kaliannan
}

(I)

Crystal data

$\mathrm{C}_{9} \mathrm{H}_{14} \mathrm{NO}^{+} \mathrm{Br}^{-} \cdot 2 \mathrm{H}_{2} \mathrm{O}$

$F(000)=552$

$M_{r}=268.15$

Monoclinic, $P 2_{1} / c$

Hall symbol: -P $2 \mathrm{ybc}$

$D_{\mathrm{x}}=1.476 \mathrm{Mg} \mathrm{m}^{-3}$

$a=10.5747$ (3) $\AA$

$b=8.0382(1) \AA$

$c=15.0377$ (4) $\AA$

$\beta=109.298(1)^{\circ}$

Mo $K \alpha$ radiation, $\lambda=0.71073 \AA$

$V=1206.41(5) \AA^{3}$

$Z=4$

Cell parameters from 2935 reflections

$\theta=1.0-27.5^{\circ}$

$\mu=3.39 \mathrm{~mm}^{-1}$

$T=120 \mathrm{~K}$

Block, colourless

$0.54 \times 0.48 \times 0.12 \mathrm{~mm}$

\section{Data collection}

Nonius KappaCCD diffractometer

Radiation source: Bruker Nonius FR591 rotating anode

$10 \mathrm{~cm}$ confocal mirrors monochromator

$\varphi$ and $\omega$ scans

Absorption correction: multi-scan

(SADABS; Sheldrick, 2003)

$T_{\min }=0.176, T_{\max }=0.666$

15899 measured reflections

2768 independent reflections

2455 reflections with $I>2 \sigma(I)$

$R_{\text {int }}=0.033$

$\theta_{\text {max }}=27.5^{\circ}, \theta_{\text {min }}=2.0^{\circ}$

$h=-13 \rightarrow 13$

$k=-10 \rightarrow 10$

$l=-19 \rightarrow 19$

\section{Refinement}

Refinement on $F^{2}$

Least-squares matrix: full

$R\left[F^{2}>2 \sigma\left(F^{2}\right)\right]=0.021$

$w R\left(F^{2}\right)=0.052$

$S=1.04$

2768 reflections

131 parameters

0 restraints

Primary atom site location: structure-invariant direct methods

Secondary atom site location: difference Fourier map

Hydrogen site location: inferred from neighbouring sites

$\mathrm{H}$-atom parameters constrained

$w=1 /\left[\sigma^{2}\left(F_{\mathrm{o}}{ }^{2}\right)+(0.0208 P)^{2}+0.702 P\right]$

where $P=\left(F_{\mathrm{o}}{ }^{2}+2 F_{\mathrm{c}}{ }^{2}\right) / 3$

$(\Delta / \sigma)_{\max }=0.001$

$\Delta \rho_{\max }=0.30 \mathrm{e} \AA^{-3}$

$\Delta \rho_{\min }=-0.52$ e $\AA^{-3}$

Extinction correction: SHELXL, $\mathrm{Fc}^{*}=\mathrm{kFc}\left[1+0.001 \mathrm{xFc}^{2} \lambda^{3} / \sin (2 \theta)\right]^{-1 / 4}$

Extinction coefficient: 0.0104 (6) 


\section{Special details}

Geometry. All e.s.d.'s (except the e.s.d. in the dihedral angle between two 1.s. planes) are estimated using the full covariance matrix. The cell e.s.d.'s are taken into account individually in the estimation of e.s.d.'s in distances, angles and torsion angles; correlations between e.s.d.'s in cell parameters are only used when they are defined by crystal symmetry. An approximate (isotropic) treatment of cell e.s.d.'s is used for estimating e.s.d.'s involving l.s. planes.

Refinement. Refinement of $F^{2}$ against ALL reflections. The weighted $R$-factor $w R$ and goodness of fit $S$ are based on $F^{2}$, conventional $R$-factors $R$ are based on $F$, with $F$ set to zero for negative $F^{2}$. The threshold expression of $F^{2}>\sigma\left(F^{2}\right)$ is used only for calculating $R$-factors (gt) etc. and is not relevant to the choice of reflections for refinement. $R$-factors based on $F^{2}$ are statistically about twice as large as those based on $F$, and $R$ - factors based on ALL data will be even larger.

Fractional atomic coordinates and isotropic or equivalent isotropic displacement parameters $\left(\hat{A}^{2}\right)$

\begin{tabular}{lllll}
\hline & $x$ & $y$ & $z$ & $U_{\text {iss }} / U_{\text {eq }}$ \\
\hline O1 & $0.53552(11)$ & $0.11597(15)$ & $-0.12166(8)$ & $0.0210(3)$ \\
H1 & 0.6082 & 0.1578 & -0.1037 & $0.025^{*}$ \\
N1 & $0.30377(13)$ & $0.25353(16)$ & $0.03692(9)$ & $0.0151(3)$ \\
C2 & $0.25160(15)$ & $0.15705(19)$ & $-0.04183(11)$ & $0.0164(3)$ \\
C3 & $0.33045(16)$ & $0.11197(19)$ & $-0.09427(11)$ & $0.0167(3)$ \\
H3 & 0.2949 & 0.0458 & -0.1474 & $0.020^{*}$ \\
C4 & $0.46300(16)$ & $0.16421(19)$ & $-0.06866(11)$ & $0.0159(3)$ \\
C5 & $0.51367(15)$ & $0.26305(19)$ & $0.01139(11)$ & $0.0162(3)$ \\
H5 & 0.6019 & 0.2999 & 0.0295 & $0.019^{*}$ \\
C6 & $0.43404(15)$ & $0.30649(19)$ & $0.06379(11)$ & $0.0162(3)$ \\
C7 & $0.49030(18)$ & $0.4105(2)$ & $0.15046(13)$ & $0.0242(4)$ \\
H7A & 0.5830 & 0.4336 & 0.1602 & $0.036^{*}$ \\
H7B & 0.4825 & 0.3515 & 0.2039 & $0.036^{*}$ \\
H7C & 0.4415 & 0.5132 & 0.1428 & $0.036^{*}$ \\
C8 & $0.21871(17)$ & $0.2994(2)$ & $0.09484(12)$ & $0.0203(3)$ \\
H8A & 0.1264 & 0.3101 & 0.0541 & $0.024^{*}$ \\
H8B & 0.2474 & 0.4065 & 0.1243 & $0.024^{*}$ \\
C9 & $0.22680(19)$ & $0.1707(2)$ & $0.17072(13)$ & $0.0252(4)$ \\
H9A & 0.2006 & 0.0639 & 0.1419 & $0.038^{*}$ \\
H9B & 0.1678 & 0.2021 & 0.2046 & $0.038^{*}$ \\
H9C & 0.3171 & 0.1648 & 0.2135 & $0.038^{*}$ \\
C10 & $0.10880(16)$ & $0.1013(2)$ & $-0.07030(13)$ & $0.0241(4)$ \\
H1A & 0.0942 & 0.0425 & -0.0190 & $0.036^{*}$ \\
H1B & 0.0897 & 0.0292 & -0.1240 & $0.036^{*}$ \\
H1C & 0.0509 & 0.1966 & -0.0861 & $0.036^{*}$ \\
O1W & $0.22566(12)$ & $0.76473(15)$ & $0.07413(9)$ & $0.0252(3)$ \\
H11 & 0.2091 & 0.7218 & 0.1186 & $0.030^{*}$ \\
H12 & 0.1964 & 0.7154 & 0.0286 & $0.030^{*}$ \\
O2W & $0.15723(11)$ & $0.63640(15)$ & $0.21883(8)$ & $0.0221(3)$ \\
H21 & 0.1498 & 0.7078 & 0.2552 & $0.027^{*}$ \\
H22 & 0.0903 & 0.5849 & 0.2026 & $0.027^{*}$ \\
Br1 & $0.123005(16)$ & $0.92168(2)$ & $0.370123(12)$ & $0.02309(7)$ \\
& & & &
\end{tabular}


Atomic displacement parameters $\left(\AA^{2}\right)$

\begin{tabular}{lllllll}
\hline & $U^{11}$ & $U^{22}$ & $U^{33}$ & $U^{12}$ & $U^{13}$ & $U^{23}$ \\
\hline O1 & $0.0193(6)$ & $0.0291(6)$ & $0.0176(6)$ & $-0.0035(5)$ & $0.0102(5)$ & $-0.0053(5)$ \\
N1 & $0.0185(6)$ & $0.0149(6)$ & $0.0138(7)$ & $0.0036(5)$ & $0.0079(5)$ & $0.0023(5)$ \\
C2 & $0.0168(7)$ & $0.0171(7)$ & $0.0136(8)$ & $0.0022(6)$ & $0.0029(6)$ & $0.0045(6)$ \\
C3 & $0.0193(8)$ & $0.0177(7)$ & $0.0121(8)$ & $-0.0003(6)$ & $0.0038(6)$ & $0.0002(6)$ \\
C4 & $0.0189(8)$ & $0.0164(7)$ & $0.0137(8)$ & $0.0025(6)$ & $0.0072(6)$ & $0.0024(6)$ \\
C5 & $0.0164(7)$ & $0.0182(8)$ & $0.0141(8)$ & $-0.0018(6)$ & $0.0051(6)$ & $0.0009(6)$ \\
C6 & $0.0191(8)$ & $0.0141(7)$ & $0.0149(8)$ & $0.0008(6)$ & $0.0048(6)$ & $0.0021(6)$ \\
C7 & $0.0297(9)$ & $0.0252(9)$ & $0.0193(9)$ & $-0.0042(7)$ & $0.0100(7)$ & $-0.0056(7)$ \\
C8 & $0.0238(8)$ & $0.0200(8)$ & $0.0225(9)$ & $0.0047(7)$ & $0.0148(7)$ & $0.0013(7)$ \\
C9 & $0.0330(10)$ & $0.0243(9)$ & $0.0243(10)$ & $0.0016(7)$ & $0.0177(8)$ & $0.0025(7)$ \\
C10 & $0.0166(8)$ & $0.0306(9)$ & $0.0245(10)$ & $-0.0001(7)$ & $0.0060(7)$ & $0.0021(7)$ \\
O1W & $0.0263(6)$ & $0.0302(6)$ & $0.0224(7)$ & $-0.0068(5)$ & $0.0125(5)$ & $-0.0044(5)$ \\
O2W & $0.0223(6)$ & $0.0229(6)$ & $0.0212(6)$ & $-0.0018(5)$ & $0.0071(5)$ & $-0.0026(5)$ \\
Br1 & $0.02279(10)$ & $0.02429(11)$ & $0.02064(11)$ & $0.00401(7)$ & $0.00508(7)$ & $-0.00067(7)$ \\
& & & & & & \\
\hline
\end{tabular}

Geometric parameters $\left(\AA,{ }^{\circ}\right)$

\begin{tabular}{|c|c|c|c|}
\hline $\mathrm{O} 1-\mathrm{C} 4$ & $1.3332(18)$ & $\mathrm{C} 7-\mathrm{H} 7 \mathrm{C}$ & 0.960 \\
\hline $\mathrm{O} 1-\mathrm{H} 1$ & 0.800 & $\mathrm{C} 8-\mathrm{C} 9$ & $1.522(2)$ \\
\hline N1-C6 & $1.369(2)$ & $\mathrm{C} 8-\mathrm{H} 8 \mathrm{~A}$ & 0.970 \\
\hline $\mathrm{N} 1-\mathrm{C} 2$ & $1.370(2)$ & $\mathrm{C} 8-\mathrm{H} 8 \mathrm{~B}$ & 0.970 \\
\hline $\mathrm{N} 1-\mathrm{C} 8$ & $1.4905(19)$ & C9-H9A & 0.960 \\
\hline $\mathrm{C} 2-\mathrm{C} 3$ & $1.372(2)$ & C9-H9B & 0.960 \\
\hline $\mathrm{C} 2-\mathrm{C} 10$ & $1.496(2)$ & $\mathrm{C} 9-\mathrm{H} 9 \mathrm{C}$ & 0.960 \\
\hline $\mathrm{C} 3-\mathrm{C} 4$ & $1.390(2)$ & $\mathrm{C} 10-\mathrm{H} 1 \mathrm{~A}$ & 0.960 \\
\hline $\mathrm{C} 3-\mathrm{H} 3$ & 0.930 & $\mathrm{C} 10-\mathrm{H} 1 \mathrm{~B}$ & 0.960 \\
\hline $\mathrm{C} 4-\mathrm{C} 5$ & $1.393(2)$ & $\mathrm{C} 10-\mathrm{H} 1 \mathrm{C}$ & 0.960 \\
\hline $\mathrm{C} 5-\mathrm{C} 6$ & $1.375(2)$ & $\mathrm{O} 1 \mathrm{~W}-\mathrm{H} 11$ & 0.821 \\
\hline $\mathrm{C} 5-\mathrm{H} 5$ & 0.930 & $\mathrm{O} 1 \mathrm{~W}-\mathrm{H} 12$ & 0.763 \\
\hline $\mathrm{C} 6-\mathrm{C} 7$ & $1.496(2)$ & $\mathrm{O} 2 \mathrm{~W}-\mathrm{H} 21$ & 0.815 \\
\hline $\mathrm{C} 7-\mathrm{H} 7 \mathrm{~A}$ & 0.960 & $\mathrm{O} 2 \mathrm{~W}-\mathrm{H} 22$ & 0.786 \\
\hline $\mathrm{C} 7-\mathrm{H} 7 \mathrm{~B}$ & 0.960 & & \\
\hline $\mathrm{C} 4-\mathrm{O} 1-\mathrm{H} 1$ & 110.7 & $\mathrm{H} 7 \mathrm{~A}-\mathrm{C} 7-\mathrm{H} 7 \mathrm{C}$ & 109.5 \\
\hline $\mathrm{C} 6-\mathrm{N} 1-\mathrm{C} 2$ & $120.65(13)$ & $\mathrm{H} 7 \mathrm{~B}-\mathrm{C} 7-\mathrm{H} 7 \mathrm{C}$ & 109.5 \\
\hline $\mathrm{C} 6-\mathrm{N} 1-\mathrm{C} 8$ & $119.56(13)$ & $\mathrm{N} 1-\mathrm{C} 8-\mathrm{C} 9$ & $112.01(13)$ \\
\hline $\mathrm{C} 2-\mathrm{N} 1-\mathrm{C} 8$ & $119.79(13)$ & $\mathrm{N} 1-\mathrm{C} 8-\mathrm{H} 8 \mathrm{~A}$ & 109.2 \\
\hline $\mathrm{N} 1-\mathrm{C} 2-\mathrm{C} 3$ & $119.91(14)$ & $\mathrm{C} 9-\mathrm{C} 8-\mathrm{H} 8 \mathrm{~A}$ & 109.2 \\
\hline $\mathrm{N} 1-\mathrm{C} 2-\mathrm{C} 10$ & $119.81(14)$ & $\mathrm{N} 1-\mathrm{C} 8-\mathrm{H} 8 \mathrm{~B}$ & 109.2 \\
\hline $\mathrm{C} 3-\mathrm{C} 2-\mathrm{C} 10$ & $120.28(15)$ & $\mathrm{C} 9-\mathrm{C} 8-\mathrm{H} 8 \mathrm{~B}$ & 109.2 \\
\hline $\mathrm{C} 2-\mathrm{C} 3-\mathrm{C} 4$ & $120.64(15)$ & $\mathrm{H} 8 \mathrm{~A}-\mathrm{C} 8-\mathrm{H} 8 \mathrm{~B}$ & 107.9 \\
\hline $\mathrm{C} 2-\mathrm{C} 3-\mathrm{H} 3$ & 119.7 & $\mathrm{C} 8-\mathrm{C} 9-\mathrm{H} 9 \mathrm{~A}$ & 109.5 \\
\hline $\mathrm{C} 4-\mathrm{C} 3-\mathrm{H} 3$ & 119.7 & $\mathrm{C} 8-\mathrm{C} 9-\mathrm{H} 9 \mathrm{~B}$ & 109.5 \\
\hline $\mathrm{O} 1-\mathrm{C} 4-\mathrm{C} 3$ & $118.23(14)$ & $\mathrm{H} 9 \mathrm{~A}-\mathrm{C} 9-\mathrm{H} 9 \mathrm{~B}$ & 109.5 \\
\hline $\mathrm{O} 1-\mathrm{C} 4-\mathrm{C} 5$ & $123.32(14)$ & $\mathrm{C} 8-\mathrm{C} 9-\mathrm{H} 9 \mathrm{C}$ & 109.5 \\
\hline
\end{tabular}




$\begin{array}{ll}\mathrm{C} 3-\mathrm{C} 4-\mathrm{C} 5 & 118.44(14) \\ \mathrm{C} 6-\mathrm{C} 5-\mathrm{C} 4 & 120.43(14) \\ \mathrm{C} 6-\mathrm{C} 5-\mathrm{H} 5 & 119.8 \\ \mathrm{C} 4-\mathrm{C} 5-\mathrm{H} 5 & 119.8 \\ \mathrm{~N} 1-\mathrm{C} 6-\mathrm{C} 5 & 119.93(14) \\ \mathrm{N} 1-\mathrm{C} 6-\mathrm{C} 7 & 120.17(14) \\ \mathrm{C} 5-\mathrm{C} 6-\mathrm{C} 7 & 119.90(14) \\ \mathrm{C} 6-\mathrm{C} 7-\mathrm{H} 7 \mathrm{~A} & 109.5 \\ \mathrm{C} 6-\mathrm{C} 7-\mathrm{H} 7 \mathrm{~B} & 109.5 \\ \mathrm{H} 7 \mathrm{~A}-\mathrm{C} 7-\mathrm{H} 7 \mathrm{~B} & 109.5 \\ \mathrm{C} 6-\mathrm{C} 7-\mathrm{H} 7 \mathrm{C} & 109.5 \\ & \\ \mathrm{C} 6-\mathrm{N} 1-\mathrm{C} 2-\mathrm{C} 3 & 0.3(2) \\ \mathrm{C} 8-\mathrm{N} 1-\mathrm{C} 2-\mathrm{C} 3 & -178.74(14) \\ \mathrm{C} 6-\mathrm{N} 1-\mathrm{C} 2-\mathrm{C} 10 & -179.64(14) \\ \mathrm{C} 8-\mathrm{N} 1-\mathrm{C} 2-\mathrm{C} 10 & 1.3(2) \\ \mathrm{N} 1-\mathrm{C} 2-\mathrm{C} 3-\mathrm{C} 4 & -0.4(2) \\ \mathrm{C} 10-\mathrm{C} 2-\mathrm{C} 3-\mathrm{C} 4 & 179.54(15) \\ \mathrm{C} 2-\mathrm{C} 3-\mathrm{C} 4-\mathrm{O} 1 & 179.55(14) \\ \mathrm{C} 2-\mathrm{C} 3-\mathrm{C} 4-\mathrm{C} 5 & 0.1(2) \\ \mathrm{O} 1-\mathrm{C} 4-\mathrm{C} 5-\mathrm{C} 6 & -179.05(14)\end{array}$

$\begin{array}{ll}\text { H9A-C9-H9C } & 109.5 \\ \text { H9B-C9-H9C } & 109.5 \\ \text { C2-C10-H1A } & 109.5 \\ \text { C2-C10-H1B } & 109.5 \\ \text { H1A-C10-H1B } & 109.5 \\ \text { C2-C10-H1C } & 109.5 \\ \text { H1A-C10-H1C } & 109.5 \\ \text { H1B-C10-H1C } & 109.5 \\ \text { H11-O1W-H12 } & 112.4 \\ \text { H21-O2W-H22 } & 107.6\end{array}$

$\mathrm{C} 3-\mathrm{C} 4-\mathrm{C} 5-\mathrm{C} 6 \quad 0.4(2)$

$\mathrm{C} 2-\mathrm{N} 1-\mathrm{C} 6-\mathrm{C} 5 \quad 0.1(2)$

$\mathrm{C} 8-\mathrm{N} 1-\mathrm{C} 6-\mathrm{C} 5 \quad 179.21(14)$

$\mathrm{C} 2-\mathrm{N} 1-\mathrm{C} 6-\mathrm{C} 7 \quad-179.43(14)$

$\mathrm{C} 8-\mathrm{N} 1-\mathrm{C} 6-\mathrm{C} 7 \quad-0.4(2)$

$\mathrm{C} 4-\mathrm{C} 5-\mathrm{C} 6-\mathrm{N} 1 \quad-0.5(2)$

$\mathrm{C} 4-\mathrm{C} 5-\mathrm{C} 6-\mathrm{C} 7 \quad 179.06(15)$

$\mathrm{C} 6-\mathrm{N} 1-\mathrm{C} 8-\mathrm{C} 9 \quad-90.24(18)$

$\mathrm{C} 2-\mathrm{N} 1-\mathrm{C} 8-\mathrm{C} 9 \quad 88.85(18)$

Hydrogen-bond geometry $\left(A,{ }^{\circ}\right)$

\begin{tabular}{lllll}
\hline$D-\mathrm{H} \cdots A$ & $D-\mathrm{H}$ & $\mathrm{H} \cdots A$ & $D \cdots A$ & $D-\mathrm{H} \cdots A$ \\
\hline $\mathrm{O} 1-\mathrm{H} 1 \cdots \mathrm{O} 1 W^{\mathrm{i}}$ & 0.80 & 1.78 & $2.5720(16)$ & 173 \\
$\mathrm{O} 1 W-\mathrm{H} 11 \cdots \mathrm{O} 2 W$ & 0.82 & 1.90 & $2.7135(16)$ & 175 \\
$\mathrm{O} 1 W-\mathrm{H} 12 \cdots \mathrm{Br} 1^{\mathrm{ii}}$ & 0.76 & 2.50 & $3.2610(12)$ & 172 \\
$\mathrm{O} 2 W-\mathrm{H} 21 \cdots \mathrm{Br} 1$ & 0.81 & 2.52 & $3.3332(12)$ & 178 \\
$\mathrm{O} 2 W-\mathrm{H} 22 \cdots \mathrm{Br} 1^{\mathrm{iii}}$ & 0.79 & 2.52 & $3.3061(12)$ & 173
\end{tabular}

Symmetry codes: (i) $-x+1,-y+1,-z$; (ii) $x,-y+3 / 2, z-1 / 2$; (iii) $-x, y-1 / 2,-z+1 / 2$. 